\title{
Negative Refractive Index Metamaterial Structure Using SRR by Incidenting the Light Horizontally
}

\author{
Ammar Armghan, Xinguang Hu, Shuai Yuan, Jinsong Xia* \\ Wuhan National Laboratory for Optoelectronics and School of Optical and Electronic Information, Huazhong \\ University of Science and Technology, Wuhan, China \\ Email:ammar.armghan@gmail.com, ${ }^{*}$ jsxia@hust.edu.cn
}

Received 19 October 2015; accepted 15 November 2015; published 18 November 2015

Copyright (C) 2015 by authors and Scientific Research Publishing Inc.

This work is licensed under the Creative Commons Attribution International License (CC BY). http://creativecommons.org/licenses/by/4.0/

c) (i) Open Access

\begin{abstract}
Metamaterial structure based on split ring resonators (SRR) is proposed in order to produce a negative refractive index. For this structure we have used a new approach, instead of applying light perpendicularly incident. We apply horizontally incident input waves. A model of SRR is used to understand the behavior and its affects. We calculate the $S$-parameters using $S$-parameter analysis and the results for transmission, refractive index, permeability and permittivity of the structure is induced. The negative refractive index is found to be significantly dependent upon the width of the continuous wire as well as gap between resonators. Moreover, we study the effect of lattice constant on the electromagnetic response of the structure. It is expected that this work will provide useful information for design and fabrication of metamaterials with negative refractive index for in-plane applications.
\end{abstract}

\section{Keywords}

Metamaterials, Negative Refractive Index, Left Handed Material, Scattering Parameters

\section{Introduction}

Metamaterial is a composite structure made of metals and dielectrics. It implies that it is not a homogenous material, rather inhomogeneous artificial materials made of metal and dielectric materials. Metallic or dielectric structures in metamaterials are typically small; they are actually much smaller than the wavelength. Because of which, at a wavelength scale, we can assume an effectively homogenous material but with very different physi-

"Corresponding author.

How to cite this paper: Armghan, A., Hu, X.G., Yuan, S. and Xia, J.S. (2015) Negative Refractive Index Metamaterial Structure Using SRR by Incidenting the Light Horizontally. Journal of Electromagnetic Analysis and Applications, 7, $276-282$.

http://dx.doi.org/10.4236/jemaa.2015.711029 
cal properties in comparison to either metals or dielectrics. So, now we are creating artificial materials made of metallic and dielectric materials in a way that they have the desired property. The first quadrant has a positive $\varepsilon$ and $\mu$, this kind of materials is called double positive materials or natural dielectric materials. If an electromagnetic wave propagates in such a material, you will see a propagating wave with some loss or attenuation. In case of metals, the $\varepsilon$ is negative and $\mu$ is positive, thus metals are also called epsilon negative materials. If electromagnetic wave propagates in a metal, it experiences huge attenuation that is why when discussing metals we discuss the skin depth effect of metal. We know that actually the waves will attenuate a lot into the metamaterials, so the actual skin depth effect for most of the electromagnetic waves is very small. In 1960s, Veselog theoretically proposed that materials having negative $\mathrm{Mu}$ and negative epsilon at the same time could produce a negative index of refraction and called them a left handed material [1]. In 1996, J. B. Pendry proposed that we could have a negative epsilon by arranging the metal wires in a simple cubic lattice. But the question still remained how we could get the negative Mu that was answered in 2000 by smith [2] [3]. Smith proposed a structure consisting of periodic array of split ring resonators and continuous wires that produced the negative permeability and negative permittivity [3].

In recent times, there has been massive development in the field of metamaterials. Negative refractive index (NRI) is the main focus point of current research. Within five years after Smith's proposal, the field of NRI has seen development at optical range by using different structures like paired nano-rods [4], nano-fishnet with circular voids [5], nano-fishnet with elliptical voids [6], and nano-fishnet with rectangular voids [7].

Metamaterials in THz regime are being used as absorbers [8], quarter waveplates [9], switches and modulators [10]-[15]. Metamaterial negative refractive index has numerous applications such as M-NRI for antennas [16], Superlens [17], and wireless power transfer [18] and for biomedical applications [19]-[26].

In this paper, we propose a novel structure of metamaterials, unit cell of complementary split ring resonators at $10 \mathrm{THz}$ frequency. This structure has been simulated by FDTD methods and the S-parameters. Then we derive the parameters i.e. refractive index, permittivity and permeability by using the S-parameters analysis technique discussed by smith [5]. The simulation results shows that by incidenting the light horizontally, negative $\mathrm{Mu}$ and negative epsilon are obtained for the proposed structure, which has the properties of Double Negative (DNG) metamaterials.

Most of the current metamaterials research is that light incident on the normal direction. But many researchers are trying to integrate devices with different function on single chip. Therefore, metamaterials to work with light in plane propagation direction is necessary for chip integration. However, few research of in plane light propagation is reported in case of metamaterials. In this paper, it studies a metamaterial structure for an in plane light propagation and studies its optical properties.

\section{Design and Simulation}

\subsection{S-Parameter Extraction Technique}

Scatter parameters (S-parameters), is usually use to describe the optical behavior of structures. S-parameters relate the incident light to reflected or transmitted light. The S-parameters are complex value numbers that represent reflection and transmission coefficients. In case of metamaterials, the S-parameters are directly proportional to the electric fields. So, we simply run the simulation and look at the S-parameters as transmitted filed divided by the incident field in direction and reflected field divided by the incident field in other direction.

Ideally, the fields would be recorded on the edge of the unit cell. In practice, the measurements are usually done at some distance from the metamaterial structure. Therefore, it is necessary to compensate the phase accumulated between metamaterial structure and measurement points. Different kinds of techniques have been developed in the past few years for extraction of S-parameters. In our simulation we are using the S-parameters extraction technique discussed by Smith et al. [3].

\subsection{Simulation Technique}

The structure was simulated FDTD method. Choosing the frequency unit in THz, S-parameter analysis tool was used to extract the S-parameters. Each calculation is based on 100 frequency samples.

The unit cell is shown in Figure 1. It consists of two materials: glass substrate and split ring resonator made of copper. This structure was simulated for $10 \mathrm{THz}$ frequency range. The thickness of the substrate is $250 \mathrm{~nm}$, 


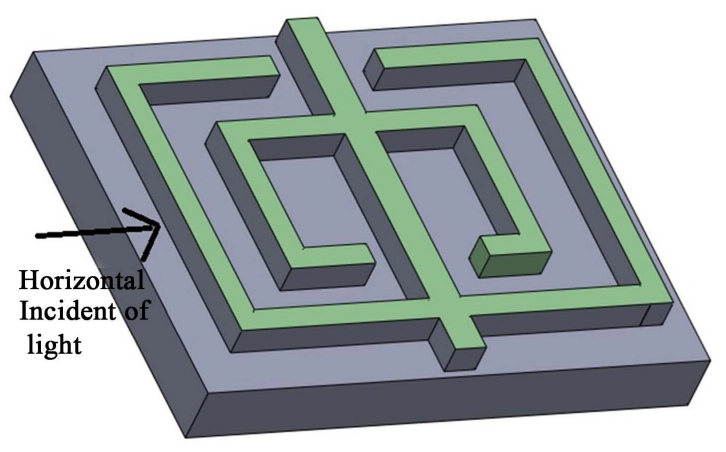

(a)

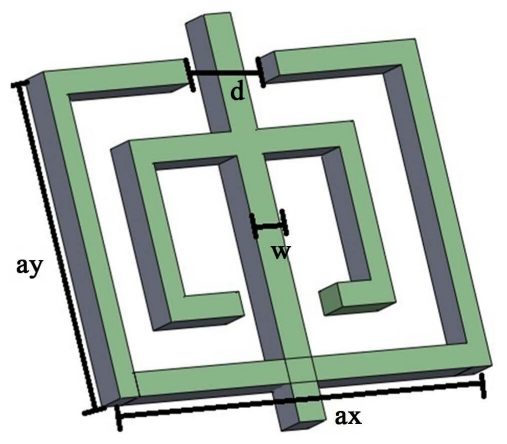

(b)

Figure 1. (a) Shows the direction of the incident light and (b) shows the parameters.

width of metal wires is $140 \mathrm{~nm}$, length of the metal wires of the outer ring is $2000 \mathrm{~nm}$ and the gap between the inner ring and the outer ring is $147 \mathrm{~nm}$.

\section{Results and Discussion}

The calculated transmission spectra of the structure is shown in Figure 2(a), where the width of the wires are kept at $140 \mathrm{~nm}$. As it can be seen in the figure, there exists two pass bands in the transmission spectra of the structure that are separated by a certain amount of frequency. The first transmission peak is at approximately 9.7 THz. This indicates that this pass band exhibits LH behavior, but the other one at around $13 \mathrm{THz}$ is has RH behavior [27] [28]. The appearance of the LH behavior can also be confirmed by using the extracted effective parameters of the structure, as presented in Figures 2(b)-(d). Obviously, both the effective permeability and permittivity are negative around the first peak, consequently the refractive index has a negative value $(n<0)$. However, the peak at around $13 \mathrm{THz}$ exhibits the RH behavior because both permeability and permittivity are positive.

Moreover, there is a dependence of the structural parameters of the metal wire on the LH behavior. We found that the electromagnetic response of the structure is significantly affected by the width of the continuous wire. Figure 3 shows the transmission spectra of the compound structure by varying the width of the continuous wire. For this purpose, the width of the wire pairs is kept at $140 \mathrm{~nm}$ while the width of the continuous wire is varied from 50 to $140 \mathrm{~nm}$.

As can be seen, the transmission peak exhibiting the LH behavior is significantly improved with the decrease in width of the continuous wire. It should also be noted that the resonance is shifted with an increase in width of the continuous wire. To confirm this phenomenon, the effective permittivity, permeability and also refractive index of the structures are extracted from the scattering parameters, as shown in Figures 3(b)-(d), respectively. As expected, the plasma frequency shifts towards a lower frequency by increase in width of the continuous wire, while the magnetic response remains roughly unchanged [24]. Thus, this explains the reason for the dependence of resonance peak on width of the continuous wire. As Figure 3(d) showing, the refractive index is decreasing with the decrease in width of the continuous wire.

Another interesting result is shown in Figure 4, where the LH behavior of the structure is also intensely affected by varying the gap $d$ between the centers of the continuous wires. To study this phenomenon, the gap $d$ was varied from 500 to $700 \mathrm{~nm}$ while the lattice constants ax and ay are $2200 \mathrm{~nm}$ and $1800 \mathrm{~nm}$ respectively. The effective permittivity, permeability and refractive index are extracted from simulation results. As it can be seen in the figure, the resonance is left-shifted by varying the gap d between the centers of the continuous wire. A reduction of negative refractive index appears when increasing the gap size.

We also studied the LH behavior dependency on the lattice constant. To study the effect of the lattice constant ay, ax is fixed at $2200 \mathrm{~nm}$ while ay is varied from 1500 to $2400 \mathrm{~nm}$. The calculated effective parameters of the structure are shown in Figure 5. As ay is reduced, the resonant plasma frequency of the structure is reduced. If ay is reduced to $1500 \mathrm{~nm}$ the magnetic resonance frequency is still higher than the plasma frequency (at $10 \mathrm{THz}$ ). If ay is reduced any further than this point, the refractive index becomes positive eliminating the LH behavior of the structure. To study the effect of the lattice constant ax, ay was kept constant at $1800 \mathrm{~nm}$ while ax was varied from 2200 to $2600 \mathrm{~nm}$. The calculated effective parameters are shown in Figure 6. As ax is increased, magnetic 


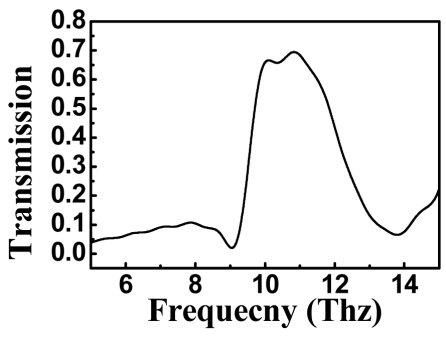

(a)

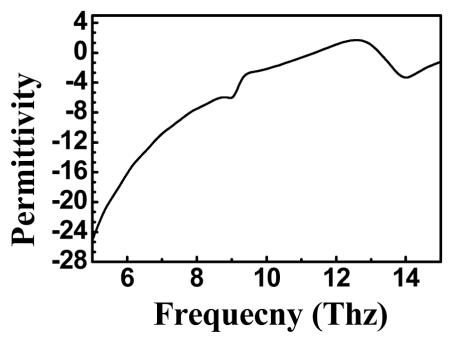

(c)

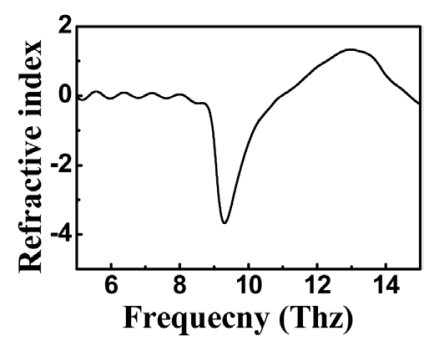

(b)

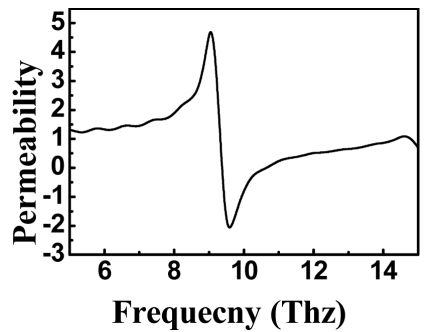

(d)

Figure 2. (a) Simulated transmission spectra of the structure, where the width of the wires are kept at $140 \mathrm{~nm}$; (b)-(d) real part of the permittivity, permeability and refractive index extracted from the simulation data, respectively.

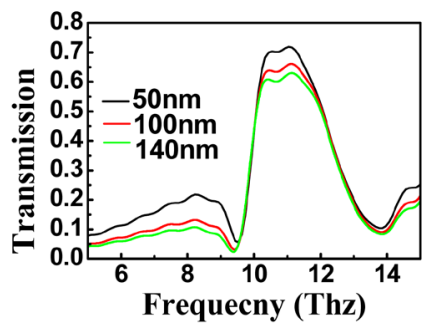

(a)

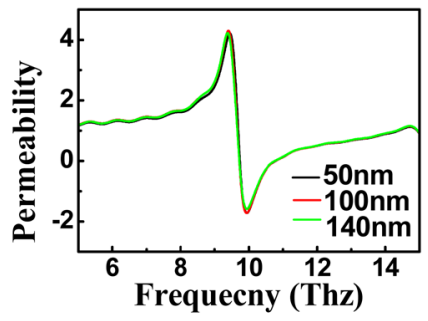

(c)

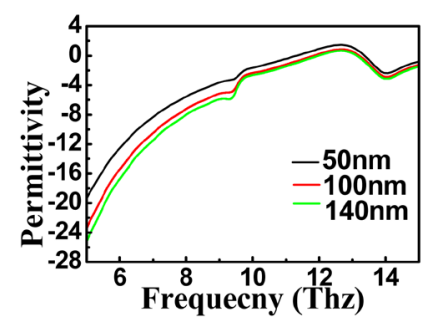

(b)

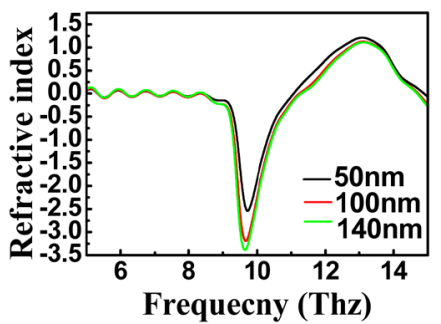

(d)

Figure 3. (a)Simulated transmission spectra of the structure with different widths of the continuous wires; (b)-(d) real part of the permittivity, permeability and refractive index, respectively.

resonance frequency of the structure is reduced while plasma frequency and the refractive index remain constant. Therefore, the refractive index is still negative.

The following Table 1 shows a comparison of this structure with other structures reported in the literature, with respect to refractive indices.

In the above table we have compared our results with the other structures that are using perpendicular direction of the incident light, where as in our design we are using the horizontal direction of incidenting light and achieve the refractive index of -3.8 . The results by using the horizontal direction of incidenting light are useful for designing novel devices for on chip integration by utilizing the optical properties of metamaterials. 


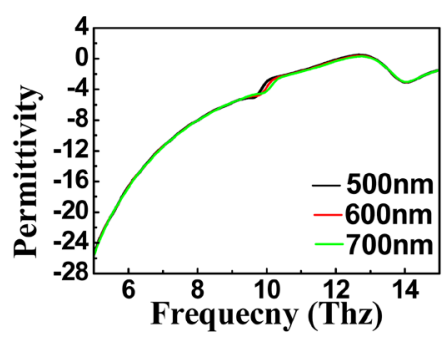

(a)

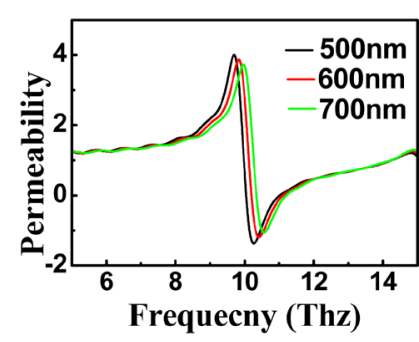

(b)

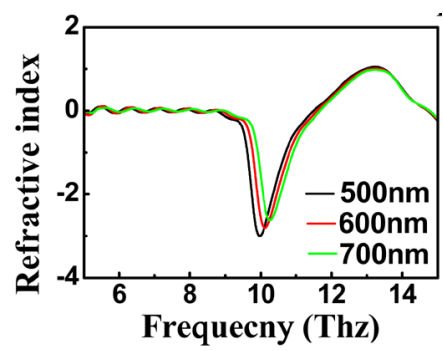

(c)

Figure 4. Extracted effective parameters of structure from the calculated scattering parameters: (a), (b) and (c) are the permittivity, permeability and refractive index, respectively. The lattice constant ax is $2200 \mathrm{~nm}$, ay is $1800 \mathrm{~nm}$.

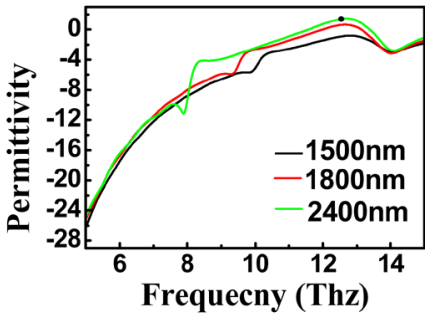

(a)

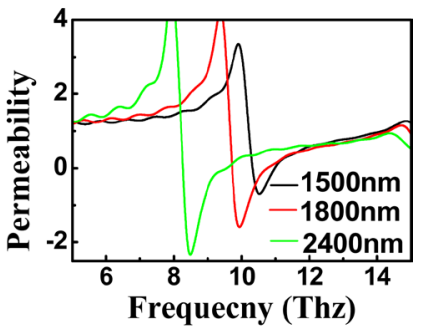

(b)

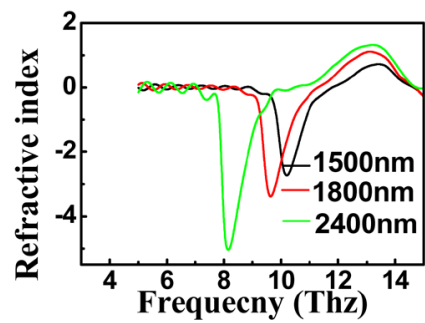

(c)

Figure 5. Extracted effective parameters of the structure from the calculated scattering parameters: (a), (b) and (c) are the permittivity, permeability and refractive index, respectively. The lattice constant ax is 2200 $\mathrm{nm}$, and ay is varied from 1500 to $2400 \mathrm{~nm}$.

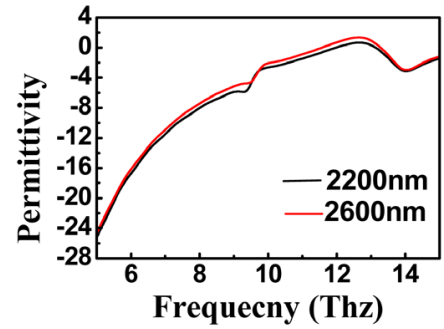

(a)

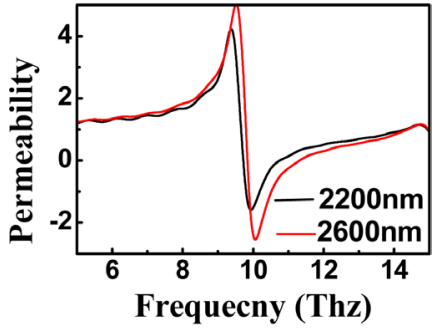

(b)

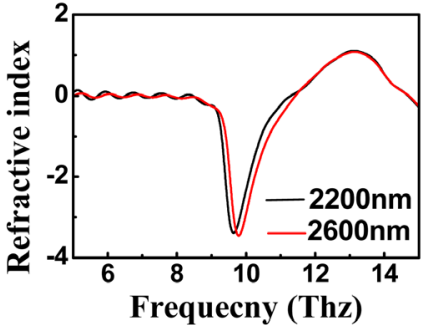

(c)

Figure 6. Extracted effective parameters of structure from the calculated scattering parameters: (a), (b) and (c) are the permittivity, permeability and refractive index, respectively. The lattice constant ay is $1800 \mathrm{~nm}$, and ax is varied from 2200 to $2600 \mathrm{~nm}$.

Table 1. Comparison between different structures.

\begin{tabular}{cccc}
\hline References & Structures & Refractive index & Direction of pump \\
\hline 4 & Paired nanorods & -0.3 & -2 \\
21 & Nano-fishnet with circular rods & -1 & Perpendicular \\
7 & Nano-fishnet with rectangular rods & -1.05 & Perpendicular \\
26 & Metal-dielectric-metal & -3.8 & Horizontally \\
Our Design & Split ring resonators & & \\
\hline
\end{tabular}

\section{Conclusion}

Simulation studies have been conducted on ring-type split ring resonators (SRR) to understand the properties of metamaterial structure with horizontally incident source. By incidenting the light along $\mathrm{x}$-axis, the proposed 
structure shows a negative refractive index indicating the properties of double negative material. Moreover, it is observed that the width of the continuous wire as well as distance between the centers affects the left-handed behavior of the structure. Additionally, influence of lattice constant on the left-handed behavior of the structure is also studied. The results indicate strong dependence of the left-handed behavior on the lattice constants ay. The search results in our paper are useful for designing novel devices for on chip integration by utilizing the optical properties of metamaterials. In subsequent works, a cascaded version of the above detailed design can be placed on top of a silicon waveguide and can be simulated to extract parameters and thus behavioral pattern of light in such environment.

\section{Acknowledgements}

This work was supported by the National “863” Program of China (No. 2015AA016904), the Major State Basic Research Development Program of China (grant 2013CB632104 and 2013CB933303).

\section{References}

[1] Veselago, V.G. (1968) The Electrodynamics of Substances with Simultaneously Negative Values of $\varepsilon$ and $\mu$. Soviet Physics Uspekhi, 10, 509-514. http://www.turpion.org/php/full/infoFT.phtml?journal_id=pu\&paper_id=3699 http://dx.doi.org/10.1070/PU1968v010n04ABEH003699

[2] Pendry, J.B., Holden, A.J. Stewart, W.J. and Youngs, I. (1996) Extremely Low Frequency Plasmonics in Metallic Mesostructures. Physical Review Letters, 76, 4773-4776. http://dx.doi.org/10.1103/PhysRevLett.76.4773

[3] Smith, D.R., Padilla, W.J., Vier, D.C., Nemat-Nasser, S.C. and Schultz, S. (2000) Composite Medium with Simultaneously Negative Permeability and Permittivity. Physical Review Letters, 84, 4184-4187. http://dx.doi.org/10.1103/PhysRevLett.84.4184

[4] Shalaev, V.M., Cai, W., Chettiar, U., Yuan, H.-K., Sarychev, A.K., Drachev, V.P. and Kildishev, A.V. (2005) Negative Index of Refraction in Optical Metamaterials. Optics Letters, 30, 3356-3358.

[5] Smith, D.R., Vier, D.C., Koschny, T. and Soukoulis, C.M. (2005) Electromagnetic Parameter Retrieval from Inhomogeneous Metamaterials. Physical Review Letters, 71, 1-11.

[6] Zhang, S., Fan, W.J., Malloy, K.J., Brueck, S.R.J., Panoiu, N.C. and Osgood, R.M. (2006) Demonstration of Metal-Dielectric Negative-Index Metamaterials with Improved Performance at Optical Frequencies. Journal of the Optical Society of America, 23, 434-438. http://dx.doi.org/10.1364/JOSAB.23.000434

[7] Dolling, G., Enkrich, C. and Wegener, M. (2006) Low-Loss Negative-Index Metamaterial at Telecommunication Wavelengths. Journal of the Optical Society of America, 31, 1800-1802.

[8] Tao, H., Landy, N.I., Bingham, C.M., Zhang, X., Averitt, R.D. and Padilla, W.J. (2008) A Metamaterial Absorber for the Terahertz Regime: Design, Fabrication and Characterization. Optics Express, 16, 7181-7188. http://dx.doi.org/10.1364/OE.16.007181

[9] Strikwerda, A.C., Fan, K., Tao, H., Pilon, D.V., Zhang, X. and Averitt, R.D. (2009) Comparison of Birefringent Electric Split-Ring Resonator and Meander Line Structures as Quarter-Wave Plates at Terahertz Frequencies. Optics Express, 17, 136-149. http://dx.doi.org/10.1364/OE.17.000136

[10] Chen, H.T., Padilla, W.J., Zide, J.M.O., Gossard, A.C., Taylor, A.J. and Averitt, R.D. (2006) Active Terahertz Metamaterial Devices. Nature, 444, 597-600. http://dx.doi.org/10.1038/nature05343

[11] Chen, H.T., Padilla, W.J., Cich, M.J., Azad, A.K., Averitt, R.D. and Taylor, A.J. (2009) A Metamaterial Solid-State Terahertz Phase Modulator. Nature Photonics, 3, 148-151. http://dx.doi.org/10.1038/nphoton.2009.3

[12] Chen, H.T., Palit, S., Tyler, T., Bingham, C.M., Zide, J.M.O., O’hara, J.F., et al. (2008) Hybrid Metamaterials Enable Fast Electrical Modulation of Freely Propagating Terahertz Waves. Applied Physics Letters, 93, Article ID: 091117. http://dx.doi.org/10.1063/1.2978071

[13] Chen, H.T., O’Hara, J.F., Azad, A.K. and Taylor, A.J. (2008) Experimental Demonstration of Frequency-Agile Terahertz Metamaterials. Nature Photonics, 2, 295-298. http://dx.doi.org/10.1038/nphoton.2008.52

[14] Lapine, M., Powell, D., Gorkunov, M., Shadrivov, I., Marqués, R. and Kivshar, Y. (2009) Structural Tunability in Metamaterials. Applied Physics Letters, 95, Article ID: 084105. http://dx.doi.org/10.1063/1.3211920

[15] Tao, H., Strikwerda, A.C., Fan, K., Padilla, W.J., Zhang, X. and Averitt, R.D. (2009) Reconfigurable Terahertz Metamaterials. Physical Review Letters, 103, Article ID: 147401. http://dx.doi.org/10.1103/PhysRevLett.103.147401

[16] Chen, X., Huifeng, M., Yang, X., Cheng, Q., Jiang, W.X. and Cui, T.J. (2009) X-Band High Directivity Lens Antenna Realized by Gradient Index Metamaterials. Proceedings of the Asia Pacific Microwave Conference, Singapore, 7-10 
December 2009, 793-797. http://dx.doi.org/10.1109/apmc.2009.5384269

[17] Lier, E. (2011) Metamaterial Lens Feed for Multiple Beam Antennas. United States Patent Application Publication, US 2011/0095953 A1. https://www.google.com/patents/US8576132

[18] Navarro-Cia, M., Beruete, M., Falcone, F., Sorolla, M. and Campillo, I. (2010) Antenna Directivity Enhancement Using a Metamaterial Parabolic Lens. Proceedings of the Fourth European Conference on Antennas and Propagation (EuCAP), Barcelona, 12-16 April 2010, 1-3. http://ieeexplore.ieee.org/xpl/login.jsp?tp=\&arnumber=5505200\&url=http\%3A\%2F\%2Fieeexplore.ieee.org\%2Fxpls\% 2Fabs_all.jsp\%3Farnumber\%3D5505200

[19] Zhang, X. and Liu, Z.W. (2008) Superlenses to Overcome the Diffraction Limit. Nature Materials, 7, 435-441. http://dx.doi.org/10.1038/nmat2141

[20] Tao, Y. and Wang, G. (2012) Conformal Hyperthermia of Superficial Tumor with Left-Handed Metamaterial Lens Applicator. IEEE Transactions on Biomedical Engineering, 59, 3525-3530. http://dx.doi.org/10.1109/TBME.2012.2218108

[21] Zhang, S., Fan, W.J., Panoiu, N.C., Malloy, K.J., Osgood, R.M. and Brueck, S.R.J. (2005) Experimental Demonstration of Near-Infrared Negative-Index Metamaterials. Physical Review Letters, 95, Article ID: 137404. http://dx.doi.org/10.1103/physrevlett.95.137404

[22] Dolling, G., Wegener, M., Soukoulis, C.M. and Linden, S. (2007) Negative-Index Metamaterial at $780 \mathrm{~nm}$ Wave Length. Journal of the Optical Society of America, 32, 53-55.

[23] Driscoll, T., Kim, H.T., Kim, B.J., Lee, Y.W., Jokerst, N.M., Palit, S., Smith, D.R., Di Ventra, M. and Basov, D.N. (2009) Memory Metamaterials. Science, 325, 1518-1521. http://dx.doi.org/10.1126/science.1176580

[24] Vu, D.L., Pham, V.T., Do, T.V., Nguyen, T.T., Vu, T.T.T., Le, V.H. and Lee, Y.P. (2010) The Electromagnetic Response of Different Metamaterial Structures. Advances in Natural Sciences, 1, 1-7.

[25] Xu, C. and Dong, J. (2010) Negative Refractive Index in Non-Resonance Spectrum Area. COL, 8, 1067-1070.

[26] Zhong, M. (2014) Influence of Dielectric Layer on Negative Refractive Index and Transmission of Metal-Dielectric-Metal Sandwiched Metamaterials. COL, 12, Article ID: 041601.

[27] Guven, K., Caliskan, M.D. and Ozbay, E. (2006) Experimental Observation of Left-Handed Transmission in a Bilayer Metamaterial under Normal-to-Plane Propagation. Optics Express, 14, 8685-8693. http://dx.doi.org/10.1364/OE.14.008685

[28] Lam, V.D., Kim, J.B., Lee, S.J. and Lee, Y.P. (2008) Left-Handed Behaviour of Combined and Fishnet Structures. Journal of Applied Physics, 103, Article ID: 033107. http://dx.doi.org/10.1063/1.2841726 